\title{
Monte Carlo Simulation of Polymer Chains with Excluded Volume on a Cubic Lattice. Local Overlap Model
}

\author{
Mark Naoshi KawaGUCHI \\ Center for Advanced Materials, Lawrence Berkeley Laboratory, and Department of Chemical Engineering, \\ University of California at Berkeley, Berkeley, CA 94720, U.S.A.
}

(Received December 15, 1995)

\begin{abstract}
Dynamic Monte Carlo simulations of isolated chains with excluded volume using a local overlap model produce scaling laws in good agreement with theory. Local overlap increases the local flexibility of the chain while maintaining long range excluded volume, and it avoids certain complications with two bead motions. Comparisons were made for data with and without excluded volume and for data with and without end effects. It was found that the prefactors for the dynamic scaling laws can be estimated from a single simulation of a typical chain length. Also, the dimensionless ratio $U=\tau D /\left\langle R^{2}\right\rangle$ is constant with respect to chain length, but the value contrasts with previous lattice simulations [D. E. Kranbuehl and P. H. Verdier, J. Chem. Phys., 71, 2662 (1979); P. H. Verdier and D. E. Kranbuehl, Macromolecules, 20, 1362 (1987)] where $\tau$ is the longest relaxation time, $D$ is the diffusion coefficient, and $\left\langle R^{2}\right\rangle$ is the mean squared end-to-end vector. KEY WORDS Monte Carlo Simulation / Isolated Chain / Dilute Solution / Relaxation Time / Diffusion Coefficient / Lattice Model /
\end{abstract}

A number of studies have wrestled with the proper modeling of excluded volume for isolated chains on a 3-D lattice. Verdier and Stockmayer ${ }^{1-4}$ began by modeling polymers as a series of beads connected by bonds of constant length. Each bond represents a sufficient number of monomers to constitute a statistical Kuhn segment. The mass of the monomers and hence the repulsive force of excluded volume is concentrated around the beads. While their equilibrium simulation results correctly matched theoretical predictions by Flory, ${ }^{5}$ their dynamic scaling behavior contrasted sharply with theory. Specifically their longest relaxation time and their diffusion coefficient as a function of chain length had scaling exponents of about 3 and -1.8 , respectively while scaling arguments by de Gennes ${ }^{6}$ predict 2.2 and -1 .

A detailed analysis by Hilhorst and Deutch ${ }^{7,8}$ found that the local bead moves chosen by Verdier and Stockmayer artificially slow the relaxation behavior substantially. They suggested that two bead "crankshaft motions" would eliminate the artificial constraint. A thorough investigation of two bead motions has been done by a number of authors. Early studies by Kranbuehl and Verdier ${ }^{9}$ continued to indicate an exponent of 3 for long chains and seemed to be confirmed by Romiszowski and Stockmayer. ${ }^{10}$ Although Gurler et al. ${ }^{11}$ obtained an exponent of 2.13 using $90^{\circ}$ crankshaft motions, their unusually low exponent of 1.88 for their model without excluded volume and lack of error estimates for their exponents make it difficult to draw solid conclusions. Subsequent work by Verdier and Kranbueh $1^{12}$ further determined that relaxation behavior is unaffected by lattice type but strongly depends on the local moves employed. Simulations on the three lattice types with combinations of one and two bead motions produced a relaxation exponent around 2.6. More recent work by Kranbuehl et al. ${ }^{13}$ found similar exponents with off-lattice models, and they concluded that "equilibrium scaling exponents cannot necessarily be used for dynamical scaling." It should be noted that more realistic Brownian dynamic simulations ${ }^{14}$ verify theoretical arguments, and the experimental literature supports the theory when hydrodynamic interactions are included, ${ }^{15}$ so the lattice model results remain puzzling. Since the author is not aware of a lattice model which accurately predicts not only the equilibrium properties, but dynamic properties such as the relaxation time and the diffusion coefficient, a different Monte Carlo model is employed here which allows local overlapping of beads and uses only single bead movements. One model has end effects incorporated while a second model creates phantom beads around the chain ends to eliminate end effects, and both models agree with analytical equilibrium and dynamic scaling calculations. The model algorithms and the calculations of chain statistics are discussed first. In RESULTS and DISCUSSION, comparisons with theory are made for the model with and without excluded volume. Finally, some time is spent discussing the features of the local overlap model.

\section{MODEL}

The model with end effects will be called model 1 in this paper. The polymer is modeled as a series of $N$ beads connected by fixed bonds, which lie on a simple cubic lattice. Bead $i$ is picked randomly, $i=1$ to $N$. If an end bead is chosen, $i=1$ or $N$, one of five new possible locations (Figure 1d) is randomly selected. If an interior bead is chosen and finds itself in a $90^{\circ}$ configuration (Figure 1b), then the bead may potentially move to the opposite corner, a kink jump in the language of Verdier and Stockmayer. ${ }^{1-4}$ For the two situations just mentioned, if the new site is unoccupied bead $i$ is automatically moved to its new location. If the proposed site is occupied by bead $i \pm 2$, bead $i$ still moves to the new location causing local overlap. If the new site is occupied by bead $i \neq i \pm 2$ then no movement occurs thus maintaining long range excluded volume. With overlap, crimped positions are possible (Figure 1c), and in such a case five new locations are possible subject to the 


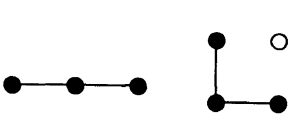

(a)

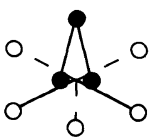

(c)

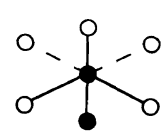

Figure 1. Local movements of the model. Local segments of the chain are shown as filled circles while potential sites for movement are denoted by open circles. A bead in a $180^{\circ}$ configuration (a) does not move. A bead in a $90^{\circ}$ (b) configuration potentially moves to the diagonally opposite corner shown. A crimped configuration (c) can occur in a model with overlapping beads, so the bead can move to one of five potential new locations. The overlapping beads are separated for clarity. For model 1, an end bead (d) can move to one of five new sites as well. For model 2, a phantom bead is attached to the end so the end bead is effectively an interior bead and undergoes the motions of (a), (b), and (c) only.

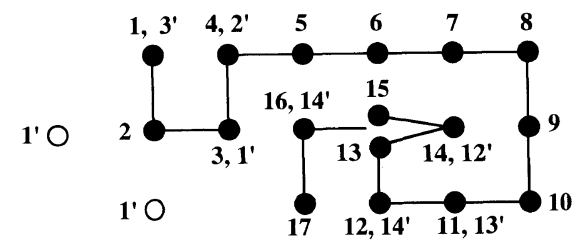

Figure 2. 2-D representation of the chain to illustrate local movements. For this 17 bead chain, new locations that satisfy the algorithm are denoted by the prime'. For the crimped position, the overlapping beads are separated for clarity.

same occupancy conditions. If an interior bead happens to be in a $180^{\circ}$ configuration (Figure 1a), the bead does not move. Model 2 is almost the same as model 1 , but it eliminates end effects by creating phantom beads 0 or $N+1$ when the computer chooses bead 1 or $N$ respectively. The end bead effectively becomes an interior bead and is subject to internal motions only (Figures 1a, 1b, 1c).

Sample movements are illustrated in the 2-D representation in Figure 2. For example, bead 5 is in a $180^{\circ}$ geometry, so it does not move. Bead 2 is an interior bead in a $90^{\circ}$ configuration. Since the bead occupying the diagonally opposite corner is a next nearest bead, $i+2$, the bead can move to position $2^{\prime}$, thus occupying the same location as bead 4 . Bead 1 , an end bead, may swivel to one of five new positions, $1^{\prime}$, including the site above and below the plane in three dimensions. One of those valid sites allows the overlap of bead 3. However, bead 4 may not flip to the opposite corner since bead 16 occupies that location. Since beads 3,5 , and 13 occupy three of five potential sites for end bead 17, it can only rotate above or below the plane. Bead 14 has a crimped geometry and can overlap next nearest neighbor beads 16 and 12 but not bead 6 . After the outcome of bead $i$ is determined, a bead cycle has passed. $N$ bead cycles should be proportional to real time, so time is measured in $N$ bead cycles for this simulation.

The mean squared end-to-end vector and the mean squared radius of gyration, $\left\langle R^{2}\right\rangle$ and $\left\langle R_{\mathrm{g}}^{2}\right\rangle$, are time averaged quantities that are easily obtained from the simulation. To determine the relaxation time, the following autocorrelation function is used:

$$
\rho(t)=\langle R(t) \cdot R(0)\rangle
$$

where $R(t)$ is the end-to-end vector at time $t$ ( $t$ is measured in $N$ bead cycles) and $\langle>$ is a time average. By plotting
Table I. Simulation and theoretical values of scaling parameters $\alpha$ and $\beta$ as a function of $N-1$ without excluded volume. Values obtained from linear regression curve fits of the form: $\ln (y)=\ln \alpha+\beta \ln (N-1)$

\begin{tabular}{ccrcc}
\hline$y$ & $\alpha$ & $\beta$ & $\alpha$ (Theory) & $\beta$ (Theory) \\
\hline$\left\langle R^{2}\right\rangle$ & $0.979 \pm 0.037$ & $1.00 \pm 0.01$ & 1 & 1 \\
$\left\langle R_{\mathrm{g}}^{2}\right\rangle$ & $0.184 \pm 0.005$ & $0.98 \pm 0.01$ & $\sim 0.167$ & 1 \\
$\tau$ & $0.124 \pm 0.022$ & $1.99 \pm 0.04$ & 0.117 & 2 \\
$D$ & $0.272 \pm 0.014$ & $-0.99 \pm 0.01$ & 0.289 & -1
\end{tabular}

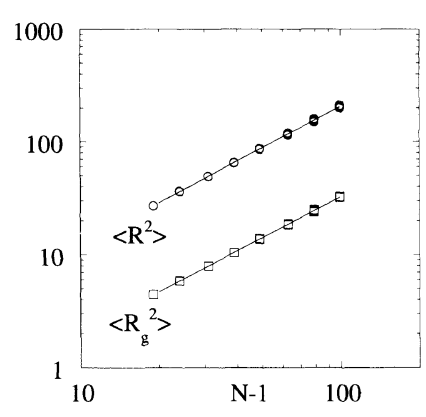

Figure 3. Log-log plot of $\left\langle R^{2}\right\rangle$ and $\left\langle R_{\mathrm{g}}^{2}\right\rangle$ versus $N-1$ where the simulation (Model 1) values are indicated by the open circles and open squares respectively. There are four data points for each chain length. The curve fits (solid lines) produce the follow power laws: $\left\langle R^{2}\right\rangle=0.739 \pm 0.009(N-1)^{1.223 \pm 0.003}$

$\left\langle R_{\mathrm{g}}^{2}\right\rangle=0.128 \pm 0.001(N-1)^{1.202 \pm 0.002}$.

$\ln \rho(t)$ versus $t$ followed by a linear regression curve fit of the linear portion at long times, the longest relaxation time $\tau$ is the negative reciprocal of the slope. The diffusion coefficient $D$ is calculated by plotting $\left\langle\left[R_{\mathrm{cm}}(t)-\right.\right.$ $\left.R_{\mathrm{cm}}(0)\right]^{2}>$ versus $t$ where $R_{\mathrm{cm}}(t)$ is the center of mass of the chain at time $t$. The diffusion coefficient is one sixth of the slope computed from linear regression. These dynamic data are collected from the simulations over times that are at least $2 \tau$ time units.

\section{RESULTS AND DISCUSSION}

The model was first run without excluded volume for $10^{6}$ time steps $\left(N \times 10^{6}\right.$ bead cycles) to compare with the analytically solvable Rouse model. ${ }^{16}$ For a simple cubic lattice the proportionality constants can be derived as well. ${ }^{17}$ For $N=20,40,60,80,100,120, \log -\log$ plots were made followed by linear regression of the form:

$$
\ln (y)=\ln \alpha+\beta \ln (N-1)
$$

where $y=\left\langle R^{2}\right\rangle,\left\langle R_{\mathrm{g}}^{2}\right\rangle, \tau$, or $D . \alpha$ is the proportionality constant and $\beta$ is the power law exponent (see Table I). The agreement between theory and simulation is good for both equilibrium and dynamic properties.

The excluded volume model (Model 1) was run 4 times for each chain length. Each simulation ran $10^{6}$ time steps for $N=20,25,32,40,50,64$, and $10^{7}$ time steps for $N=80,100$. Figure 3 shows the $\log -\log$ plots of $\left\langle R^{2}\right\rangle$ and $\left\langle R_{\mathrm{g}}^{2}\right\rangle$ as a function of chain length $N-1$. The calculated exponents $1.223 \pm 0.003$ and $1.202 \pm 0.002$ match the theoretical prediction of 1.2 rather well (see Table II). Figures 4 and 5 are $\log -\log$ plots of $\tau$ and $D$ as a function of chain length. The calculated exponents $2.226 \pm 0.016$ and $-1.005 \pm 0.008$ again closely match the theoretical predictions of 2.2 and -1 , respectively. Model 
Table II. Simulation and theoretical values of scaling parameters $\alpha$ and $\beta$ as a function of $N-1$ with excluded volume (Model 1). Values obtained from linear regression curve fits of the form: $\ln (y)=\ln \alpha+\beta \ln (N-1)$

\begin{tabular}{ccrc}
\hline$y$ & $\alpha$ & $\beta$ & $\beta$ (Theory) \\
\hline$\left\langle R^{2}\right\rangle$ & $0.739 \pm 0.009$ & $1.223 \pm 0.003$ & 1.2 \\
$\left\langle R_{\mathrm{g}}^{2}\right\rangle$ & $0.128 \pm 0.001$ & $1.202 \pm 0.002$ & 1.2 \\
$\tau$ & $0.130 \pm 0.008$ & $2.226 \pm 0.016$ & 2.2 \\
$D$ & $0.240 \pm 0.007$ & $-1.005 \pm 0.008$ & -1.0 \\
\hline
\end{tabular}

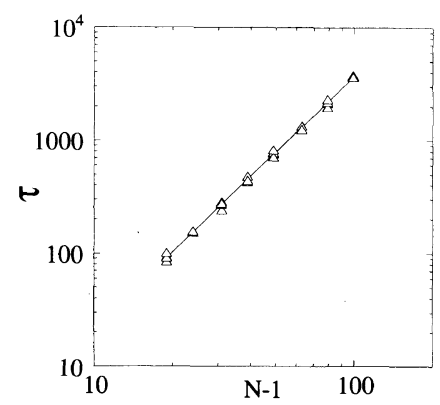

Figure 4. $\log -\log$ plot of $\tau$ versus $N-1$ with simulation data (Model 1) indicated by open triangles. There are four data points for each chain size. The resulting curve fit (solid line) is a power law: $\tau=0.130 \pm 0.008(N-1)^{2.226 \pm 0.016}$.

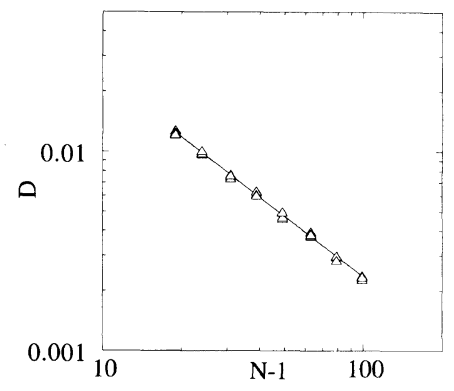

Figure 5. Log-log plot of $D$ versus $N-1$ with simulation data (Model 1) indicated by open triangles. There are 4 data points for each chain size. The resulting curve fit (solid line) is a power law: $D=0.240 \pm$ $0.007(N-1)^{-1.005 \pm 0.008}$

Table III. Simulation and theoretical values of scaling parameters $\alpha$ and $\beta$ as a function of $N-1$ with excluded volume (Model 2)

Model 2 has no end effects. Values obtained from linear regression curve fits of the form: $\ln (y)=\ln \alpha+\beta \ln (N-1)$

\begin{tabular}{ccrc}
\hline$y$ & $\alpha$ & $\beta$ & $\beta$ (Theory) \\
\hline$\left\langle R^{2}\right\rangle$ & $0.837 \pm 0.010$ & $1.202 \pm 0.003$ & 1.2 \\
$\left\langle R_{\mathrm{g}}^{2}\right\rangle$ & $0.137 \pm 0.001$ & $1.190 \pm 0.002$ & 1.2 \\
$\tau$ & $0.155 \pm 0.008$ & $2.187 \pm 0.016$ & 2.2 \\
$D$ & $0.241 \pm 0.007$ & $-1.009 \pm 0.013$ & -1.0 \\
\hline
\end{tabular}

2 was run under the same conditions with the resulting scaling laws listed in Table III. The equilibrium exponents $1.202 \pm 0.003$ and $1.190 \pm 0.002$ are somewhat smaller than Model 1 data. In the use of scaling laws, it is presumed that the proportionality constant should be a function of local constraints and dynamics only. So the increase in the coefficients in model 2 is probably caused by the reduced freedom of the end beads. The relaxation exponent is somewhat lower, $2.187 \pm 0.016$, while the diffusion exponent remains essentially the same, $-1.009 \pm 0.013$. The proportionality constants for $\tau$ and $D$ have increased and remained constant respec-
Table IV. List of probabilities of blocked moves for different local configurations for a 64 bead chain from one simulation run of $10^{6}$ time steps

\begin{tabular}{clcc}
\hline Model & \multicolumn{1}{c}{ Local structure } & $\begin{array}{c}\text { Distance } \\
\text { of move }\end{array}$ & Probability \\
\hline 1 & $90^{\circ}$ Configuration (1b) & $\sqrt{2}$ & 0.0879 \\
1 & Crimped position (1c) & $\sqrt{2}$ & 0.0972 \\
1 & Crimped position (1c) & 4 & 0.0995 \\
1 & End bead geometry (1d) & $\sqrt{2}$ & 0.0885 \\
1 & End bead geometry (1d) & 4 & 0.0943 \\
1 & All geometries & $\sqrt{2}$ and 4 & 0.0895 \\
2 & $90^{\circ}$ Configuration (1b) & $\sqrt{2}$ & 0.0847 \\
2 & Crimped position (1c) & $\sqrt{2}$ & 0.0952 \\
2 & Crimped position (1c) & 4 & 0.0978 \\
2 & All geometries & $\sqrt{2}$ and 4 & 0.0865 \\
\hline
\end{tabular}

tively. Apparently the end effects affect the relaxation time while leaving the diffusion coefficient unscathed, but the error bars are large enough to prevent any concrete conclusions.

For the excluded volume models, the proportionality constant $\alpha$ for the dynamic laws can be roughly estimated from a single simulation run by using arguments analogous to the reasoning of Evans and Edwards ${ }^{17}$ for polymer melts. Consider three possible configurations of a middle bead (Figures 1a, 1b, and 1c). These geometries occur with probability $1 / 6,2 / 3$, and $1 / 6$, respectively. For a $180^{\circ}$ configuration the bead does not move at all, but for a $90^{\circ}$ configuration it moves $\sqrt{2}$ lattice units. The bead may move $\sqrt{2}$ or 2 lattice units with weightings $4 / 5$ and $1 / 5$ in the crimped position. Define $p$ as the probability that an attempted motion does not succeed because of excluded volume, and assume further that $p$ does not depend on the local motion attempted. One can compute the expected value of the square of the monomer motion:

$$
\begin{aligned}
& \frac{1}{6}\left(0^{2}\right)+\frac{2}{3}(1-p)(\sqrt{2})^{2}+\frac{1}{6}(1-p) \\
& \times\left[\frac{4}{5}(\sqrt{2})^{2}+\frac{1}{5}(2)^{2}\right]=\frac{26}{15}(1-p)
\end{aligned}
$$

Table IV lists the excluded volume probabilities for different local configurations from one simulation run of a 64 bead chain. The probabilities in all cases are roughly similar so the one parameter assumption seems reasonable. Using the overall probability of $p=0.0895$, one can calculate the monomer diffusion coefficient and the proportionality constants:

$$
\begin{gathered}
D_{\text {mon }}=\frac{1}{6}\left(\frac{26}{15}\right)(1-p) \\
\tau=\frac{1^{2}}{3 D_{\text {mon }} \pi^{2}}(N-1)^{2.2} \\
D=D_{\text {mon }}(N-1)^{-1}
\end{gathered}
$$

For Model 1, the coefficients for $\tau$ and $D$ are 0.128 and 0.263 , respectively. These estimates are remarkably close to the simulation values. When the same analysis is done with Model 2, one gets an overall probability of 0.0865 that produces almost the same coefficients, 0.128 and 0.269 . Evan if one weights the calculation by using the 
Table V. Dimensionless scaling ratio $U=\tau D /\left\langle R^{2}\right\rangle$ as a function of $N$

$U^{0}$ corresponds to the ratio without excluded volume, $U^{1}$ is for Model 1 , and $U^{2}$ is for Model 2.

\begin{tabular}{cccc}
\hline$N$ & $U^{0}$ & $U^{1}$ & $U^{2}$ \\
\hline 20 & 0.0329 & 0.0425 & 0.0412 \\
25 & - & 0.0422 & 0.0408 \\
32 & - & 0.0410 & 0.0439 \\
40 & 0.0308 & 0.0422 & 0.0400 \\
50 & - & 0.0427 & 0.0411 \\
60 & 0.0313 & - & - \\
64 & - & 0.0429 & 0.0383 \\
80 & 0.0323 & 0.0411 & 0.0409 \\
100 & 0.0298 & 0.0423 & 0.0406 \\
120 & 0.0349 & - & - \\
Average & $0.0320 \pm 0.0018$ & $0.0421 \pm 0.0007$ & $0.0408 \pm 0.0028$ \\
\hline
\end{tabular}

specific $p$ values for each configuration, the values are about the same. Unfortunately, this analysis yields only rough estimates and cannot explain the larger relaxation proportionality constant for Model 2.

Previous authors ${ }^{18}$ have shown that the VerdierStockmayer model breaks ergodicity, so one can construct dense configurations that cannot relax at all by the local motions of the algorithm. Model 1 was tested for ergodicity by constructing a 36 bead chain that filled a $4 \times 3 \times 3$ box with the chain ends in the middle. For this situation, the Verdier-Stockmayer algorithm is unable to move at all and will always calculate an erroneous average mean squared end-to-end vector of 1 . By tracking $\left\langle R^{2}\right\rangle$ as a function of time for one simulation with Model 1, the chain rapidly relaxed to its equilibrium dimension of 57 within 1000 time steps. Thus, the model is not as constrained as the Verdier-Stockmayer model.

As others ${ }^{9,12,13}$ have done, it is interesting to calculate the dimensionless ratio:

$$
U=\frac{\tau D}{\left\langle R^{2}\right\rangle}
$$

For the Rouse model, the predicted value is the following:

$$
U^{0}=\frac{1}{3 \pi^{2}} \approx 0.0338
$$

According to scaling arguments, the dimensionless ratio should also be a constant independent of chain length even with excluded volume. Table $\mathrm{V}$ lists the values for different chain lengths with and without excluded volume. The average $U^{0}$ agrees with the predicted value within statistical error. The average $U^{1}$ (Model 1) and $U^{2}$ (Model 2) with excluded volume is significantly higher than the Rouse value. The data indicate no significant correlation between $U$ and chain length which agrees with previous lattice simulations ${ }^{12}$ but apparently contrasts with the results of off-lattice models. ${ }^{13}$ Also, the higher average value of $U$ with excluded volume in this study contradicts the lattice simulations of Verdier and Kranbuehl ${ }^{9,12}$ but agrees with the off-lattice simulations of Kranbuehl et al. ${ }^{13}$

This excluded volume model works well for several reasons. As Hilhorst and Deutch ${ }^{7}$ pointed out, the original Verdier-Stockmayer algorithm does not allow local extrema to pass each other. Local overlap eliminates
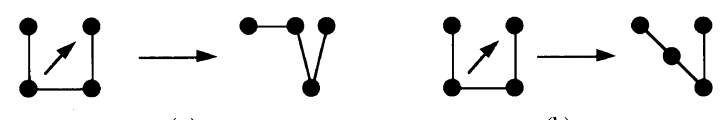

(b)

Figure 6. In (a) the local overlap excluded volume model, a corner bead flips the bead to the opposite corner overlapping with a next nearest neighbor. The beads are separated for clarity. If one imagines the bead moving "off lattice" to an intermediate location shown in (b), then the similarity to a bond fluctuation model is more clear.

the artificial constraint, and the crimped configuration provides a degree of freedom in the third dimension in a similar way that the $90^{\circ}$ crankshaft rotation does in Gurler's simulation. ${ }^{11}$ Flory ${ }^{5}$ and de Gennes ${ }^{6}$ describe excluded volume as a long range effect between chain segments far away from each other as measured along its contour length, so the scaling exponent should not be affected by changes in local constraints. Consequently, local overlap was not expected to cause a deviation from the theoretical excluded volume exponent. Also, the single bead movement scheme does not complicate the discussion that arises when two bead motions are introduced.

Upon further thought, the model presented here may be considered similar to the bond fluctuation model. Instead of actual overlap, one may imagine a bead moving "off lattice" to a position close to but not necessarily overlapping the other bead as illustrated in Figure 6 . The bonds that connect this "off lattice" bead to the rest of the chain will not lie on the lattice and will also be less than one lattice unit. Thus, bond lengths are effectively allowed to fluctuate in the local overlap model. Carmesin and Kremer $^{19}$ successfully used a bond fluctuation model to describe the dynamics of 2-D chains with excluded volume, and Shaffer ${ }^{20}$ elegantly simulated the topological effects of excluded volume in 3-D bulk melts. However, to the author's knowledge no one has applied the bond fluctuation model to isolated chains in 3-D to extract dynamic information.

\section{CONCLUSIONS}

A local overlap algorithm has been used to model dilute solution polymers on a 3-D cubic lattice with excluded volume via Monte Carlo simulation. This modification of the Verdier-Stockmayer algorithm produces equilibrium as well as dynamic scaling exponents close to the theoretical values predicted by Flory and de Gennes. The key aspect of the model lies in its ability to allow sufficient local flexibility to remove local extrema while maintaining long range excluded volume. The proportionality coefficients for the dynamic properties can be roughly estimated from one simulation, and it is not clear what influence end effects have on the local as well as global dynamics. Finally, the dimensionless scaling ratio $U$ is constant but the numerical value is significantly higher than the Rouse model prediction.

Acknowledgments. I am indebted to William W. Graessley for the discussions that laid the foundation for this work as well as Morton M. Denn and Arup K. Chakraborty for support. This work was supported in part by the Director, Office of Energy Research, Office 
of Basic Energy Sciences, Materials Science Division of the U.S. Department of Energy under Contract No. DE-AC03-76SF00098. I also wish to thank Princeton University and the University of California at Berkeley for the use of their computing facilities.

\section{REFERENCES}

1. P. H. Verdier and W. H. Stockmayer, J. Chem. Phys., 36, 227 (1962).

2. P. H. Verdier, J. Chem. Phys., 45, 2118 (1966).

3. P. H. Verdier, J. Chem. Phys., 45, 2122 (1966).

4. P. H. Verdier, J. Chem. Phys., 59, 6116 (1973).

5. P. Flory, "Principles of Polymer Chemistry," Cornell University Press, Ithaca, New York, N.Y., 1953.

6. P. G. de Gennes, "Scaling Concepts in Polymer Physics," Cornell University Press, Ithaca, New York, N.Y., 1979.

7. H. J. Hilhorst and J. M. Deutch, J. Chem. Phys., 63, 5153 (1975)

8. H. Boots and J. M. Deutch, J. Chem. Phys., 67, 4608 (1977).
9. D. E. Kranbuehl and P. H. Verdier, J. Chem. Phys., 71, 2662 (1979)

10. P. Romiszowski and W. H. Stockmayer, J. Chem. Phys., 80, 485 (1984)

11. M. T. Gurler, C. C. Crab, D. M. Dahlin, and J. Kovac, Macromolecules, 16, 398 (1983).

12. P. H. Verdier and D. E. Kranbuehl, Macromolecules, 20, 1362 (1987)

13. D. E. Kranbuehl, D. Eichinger, and P. H. Verdier, Macromolecules, 24, 2419 (1991).

14. D. Ceperly, M. H. Kalos, and J. L. Lebowitz, Macromolecules, 14, 1472 (1981).

15. S. Amelar, C. E. Eastman, R. L. Morris, M. A. Smeltzly, T. P Lodge, and E. D. von Meerwall, Macromolecules, 24, 3505 (1991).

16. P. E. Rouse, Jr., J. Chem. Phys., 21, 1972 (1953).

17. K. E. Evans and S. F. Edwards, J. Chem. Soc., Faraday Trans. 2, 77, 1891 (1981)

18. K. Kremer and K. Binder, Compt. Phys. Rep., 7, 259 (1988).

19. I. Carmesin and K. Kremer, Macromolecules, 21, 2819 (1988).

20. J. S. Shaffer, J. Chem. Phys., 101, 4205 (1994). 